\title{
Bioethanol production from woods with the aid of nanotechnology
}

- Pretreatment for enzymatic saccharification using natural structure of cellulose -

Takashi Endo

\author{
[Translation from Synthesiology, Vol.2, No.4, p.310-320 (2009)]
}

\begin{abstract}
Bioethanol production from woody biomass by enzymatic hydrolysis of cellulosic components and fermentation has attracted much attention. In this process, pretreatment is important to improve enzymatic degradability of cellulose. A milling process is one of the most effective methods for pretreatment, but its high cost has been a problem. Recently we have developed the economically feasible wet-mechanochemical process as milling pretreatment, which can unravel cellulosic components into nanoscale fibers. Thus-obtained nanofibrous product showed a high enzymatic accessibility, while keeping the cellulose crystalline structure and the lignin content. This process is based on the understanding of the nanoscopic structural characteristics of wood and cellulose.
\end{abstract}

Keywords : Bioethanol, enzymatic saccharification, pretreatment, mechanochemical treatment, nanofiber

\section{Introduction}

Recently, the interest in bioethanol as automobile fuel has risen from the perspectives of measures for global warming and energy security. In the United States and Brazil, over 20 million kiloliters per year are produced. However, since the raw materials are food biomass such as corn and sugar cane, the increased price of foods and feedstuff in competition to the mass production of bioethanol has become an issue. Therefore, it is essential to establish a technology that allows use of nonfood biomass (cellulosic biomass) such as wood, straw, pasture grass, and others. Compared to the starch biomass such as corn, the cellulosic biomass is thought to be more effective in reducing carbon gas in terms of total life cycle assessment (LCA) from the production of raw material to the use of bioethanol ${ }^{[1]}$.

In general, bioethanol is produced by fermenting the sugar obtained from the raw materials, using yeasts. Therefore, it is necessary to hydrolyze (saccharify) the cellulose in wood to glucose. Currently, enzymatic saccharification method is drawing attention. Here, the pretreatment to enhance the enzyme reactivity of wood and cellulose is important.

Milling has been known as a highly effective pretreatment for enzymatic saccharification despite its high cost. Therefore, in developing the new pretreatment technology, by reinvestigating the milling process that is known to be effective, we clarified the mechanism by which milling enhanced the enzyme saccharification property of cellulose, using some new analysis methods. Next, we developed the new technology based on the findings obtained, and were able to construct an efficient and economically feasible pretreatment technology based on the new concept.

\section{Knowing wood and cellulose}

The pretreatment technology for enzymatic saccharification that we developed is a method that utilizes the structural characteristics of wood and cellulose. Therefore, the structures of wood and cellulose will be outlined since they are important for developing this technology.

\subsection{The basis of cellulose}

The main components of wood are cellulose, hemicellulose, and lignin. The molecules of cellulose and hemicellulose are composed of sugars while lignin is a complex aromatic compound. In wood, the percentage of cellulose is highest at about $40 \sim 50 \%$. Cellulose is a biological macromolecule where the glucose is chain-linked, and the nature of cellulose in wood is an assembly of cellulose molecules called the cellulose microfibril. When the cellulose molecules are biosynthesized, they self-assemble regularly like stacked boards to form cellulose microfibrils of $3 \sim 5 \mathrm{~nm}$ width (Fig. 1, right bottom). This microfibril is the main body of the cellulose crystal, and it does not dissolve in water or ordinary organic solvents since it is extremely stable. However, the assembly forces of the cellulose molecules are the intermolecular force and the hydrogen bond that are generally considered weak. Although the component sugar is glucose, amylose in starch has different chemical and physical properties from cellulose and dissolves in hot water. Therefore, enzymatic saccharification by amylase occurs quickly, and bioethanol can easily be produced.

\subsection{Wood structure is nanostructure}

As shown in Fig. 1 right top, the "wood fiber" is composed of nanosize "cellulose microfibrils" held together using hemicellulose and lignin as adhesives, and the "wood structure" is formed by laying the vessels and tracheids that

Biomass Technology Research Center, AIST 2-2-2 Suehiro, Hiro, Kure 737-0197, Japan E-mail : t-endo@aist.go.jp

Original manuscript received September 1, 2009, Revisions received October 13, 2009, Accepted October 14, 2009 
carry water in the middle and then layering the microfibrils around them (Fig. 1 left top). The toughness of wood results from this layer structure. This is often likened to a barrel or tub. A tub is strong because the boards are arranged circularly in a vertical direction, and the hoop is used to bundle them together in a direction 90 degrees of the boards. In the wood structure, the nanosize cellulose microfibrils act like the boards and hoops of a tub, and form a highly layered nanostructure. This tough wood structure makes the pretreatment for enzymatic saccharification very difficult.

\section{Issues in conventional technology and scenario for the development of new technology}

\subsection{Acid saccharification and enzymatic saccharification}

The method for saccharification of cellulose in the wood can be divided roughly into acid and enzyme methods. Figure 2 shows the advantages and issues of the two methods. The oldest method used is the acid saccharification using sulfuric acid. Currently, large-scale bioethanol manufacturing experiments incorporating new technologies is in progress for this method. The greatest advantage of the sulfuric acid saccharification is that the reaction occurs in a short time using inexpensive sulfuric acid as the catalyst.

However, the facilities must be resistant to sulfuric acid, and the recovery and removal of sulfuric acid from the saccharified solution and waste liquid are remaining issues. While these can be solved with the advancement of engineering technology, the greatest problem is that the produced sugar is susceptible, in principle, to overdecomposition into furfural by the action of coexisting sulfuric acid ${ }^{[2]}$. When over-decomposition occurs, the yield of sugar that enables ethanol fermentation is reduced, and the product of over-decomposition even at relatively small amounts (few \%) may inhibit yeast fermentation.

On the other hand, since, in principle, no side reaction occurs in enzymatic saccharification, the yield of ethanol end product is expected to increase. The enzymatic reaction progresses at mild conditions of about $50{ }^{\circ} \mathrm{C}$, and the environmental load is low since it does not require large amounts of chemicals. The enzymes for the saccharification of cellulose are generally called cellulase. There have been over 500 kinds identified, and reactivity of some of them is affected greatly by the crystallinity and structure of cellulose. In actual enzymatic saccharification, a mixture of several enzymes including the ones for hemicellulose is used rather than a single species of enzymes.

However, there are also issues in enzymatic saccharification. Enzymatic saccharification requires much longer time compared to acid saccharification. Also, while cellulase is used in the food and textile industries, it is actually fairly expensive. As mentioned earlier, in some cases, large quantity of enzyme is required because the enzymatic reaction may be affected by the structure of cellulose.

\subsection{Pretreatment technology for enzymatic saccharification}

Since untreated wood does not react with enzymes, it is necessary to conduct the pretreatment to increase the reactivity of wood and cellulose and to promote enzymatic saccharification. According to the empirical rules of pretreatment gathered from past R\&Ds, the points considered were: to increase of surface area by breaking up the wood into small pieces; to increase reactivity by amorphization of highly crystalline cellulose; and to breakdown and

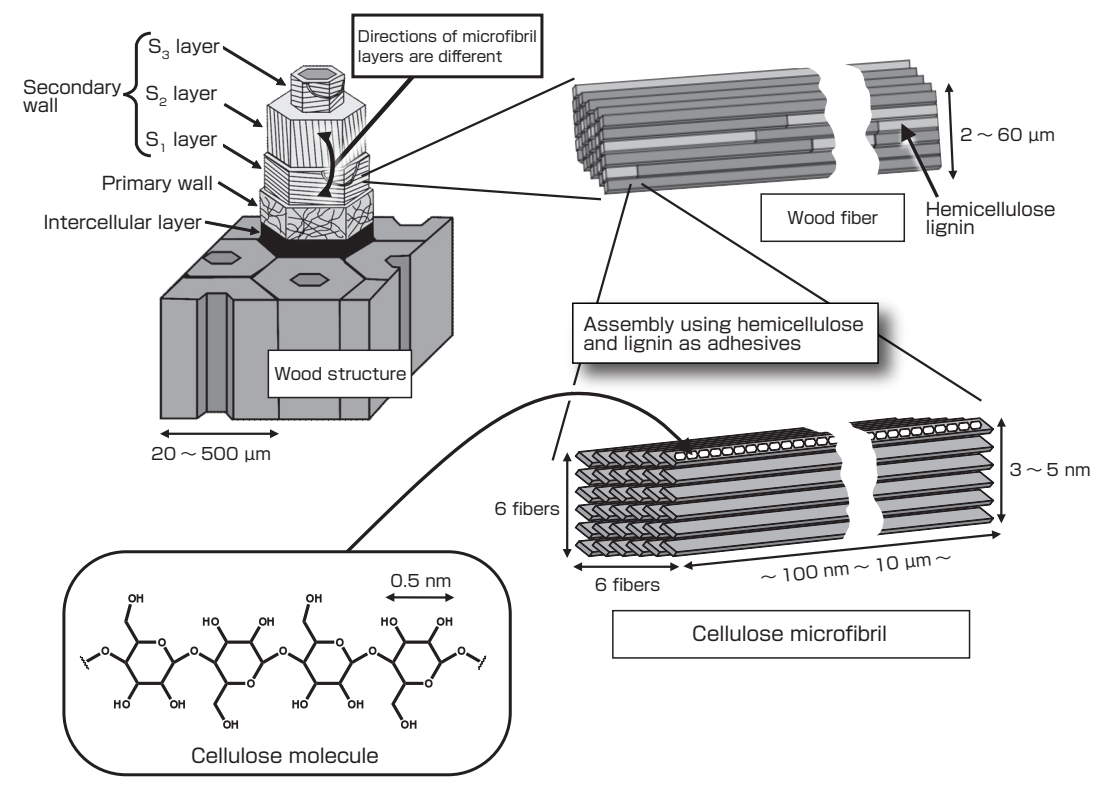

Fig. 1 Diagram of cellulose microfibril and wood structure. 
remove the lignin component that may inhibit the enzymatic activity. The pretreatments according to these rules include milling, cooking, and steam explosion ${ }^{[3]}$, but all methods have issues (table in lower part of Fig. 2). In the milling treatment, equipments such as the ball mill are used. The operation is simple and the enzymatic saccharification degree of the pretreated material is high. However, the power consumption is high and processing efficiency is low since it is batch processed. In many cases and the cost turns out high. Cooking treatment is a method where the lignin and hemicellulose in the wood are decomposed and removed using chemicals and water. It is similar to the pulping process of papermaking. Although the saccharification degree of the treated material is high, the dependency on certain wood species and the waste liquid are issues. In the steam explosion treatment, the material is placed in high-temperature high-pressure steam for a certain time, exposed to atmospheric pressure immediately, and the wood is broken down into fibers by the rapid volume expansion of steam. The need for high-pressure resistant facilities, heat recovery, and dependency on wood species are issues. Recently, hydrothermal treatment using pressurized hot water over $100{ }^{\circ} \mathrm{C}$ has drawn attention. This method uses the hydrolysis action of hot-compressed water, but like in the acid saccharification, over-decomposition tends to occur at high temperature of $200^{\circ} \mathrm{C}$ or over.

Therefore, we conducted re-experiments for the milling treatment, which is a simple yet highly effective operation, and analyzed and evaluated the mechanism for increased saccharification from the perspectives of wood chemistry and cellulose chemistry. Moreover, based on the findings, we attempted the development of the optimal pretreatment technology by combining several technologies.

\section{Mechanochemical treatment technology}

We conducted R\&D on technologies to pulverize the cellulose materials such as pulp, and technologies to composite pulp or wood powder with resin, by controlling the aggregation of particles produced by milling (control of formation of hydrogen bonds) ${ }^{[4]-[7]}$. In the process of pulverization of materials in the milling process, reactions such as breaking of chemical bonds occur. Also, the pressure and shearing force of milling can form bonds and synthesize new materials. Since milling causes the chemical reaction by the mechanical process, it is called the mechanochemical treatment. We consider the formation and breaking of bonds including weak bonds such as the hydrogen bond and the hydrophobic bond (intermolecular force) as part of the mechanochemical treatment. The mechanochemical treatment was developed originally as the technology for compositing of inorganic substances and metals, and as alloy technology. It is possible to form covalent bonds such as esterification reaction in organic substances. However, there is a "threshold value" for the change of substance to occur in milling, and breakdown and compositing will not occur even if milling is done for a long time unless enough milling energy that can set off the chemical reaction is applied.

As explained so far, the mechanochemical treatment is a relatively simple mechanical process, and has been known to improve the enzymatic saccharification of cellulose ${ }^{[8]}$. The important points were considered to be milling the wood

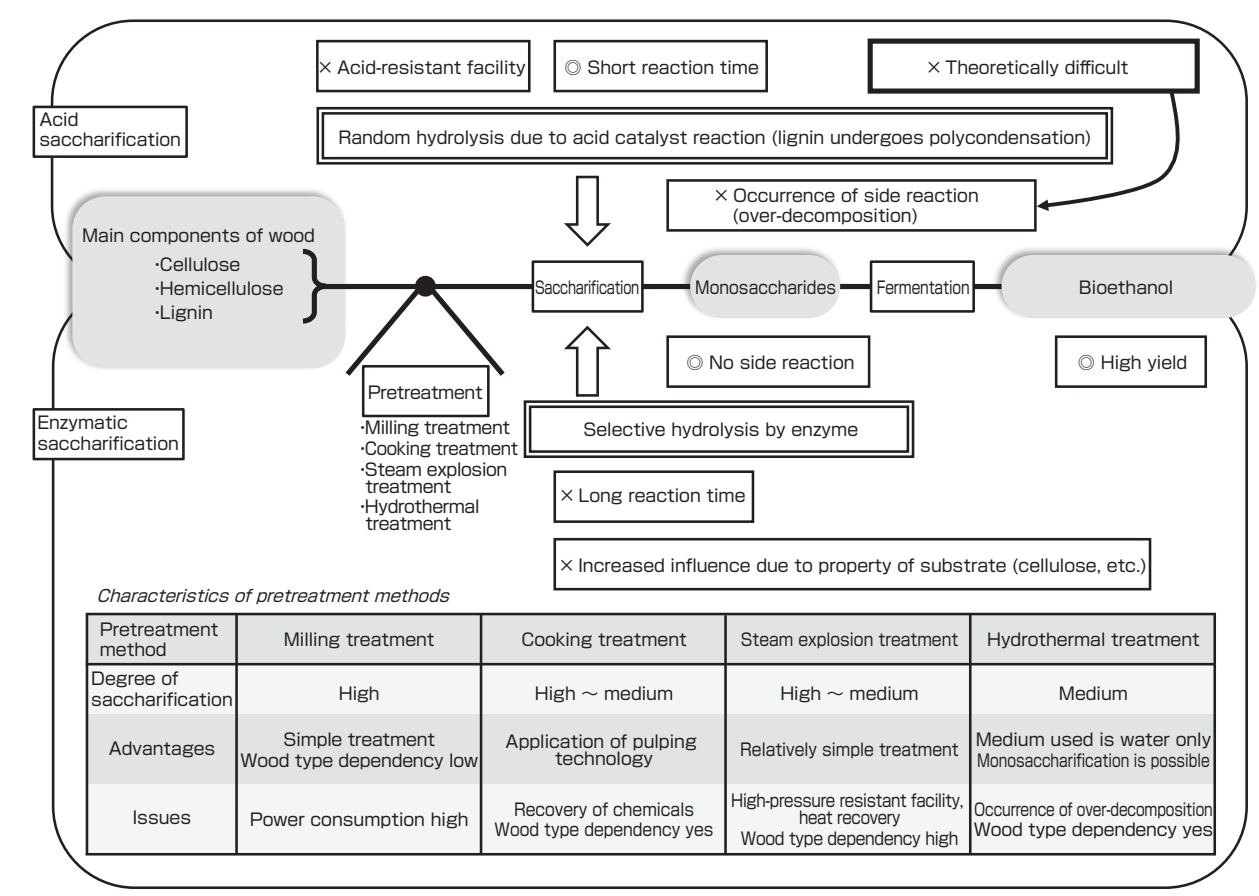

Fig. 2 Characteristics of acid saccharification and enzymatic saccharification methods. 
materials thoroughly in the dry state using equipments such as the ball mill to produce fine wood powder, and to destroy the crystal structure of cellulose (amorphization).

Consideration of the mechanism for improving the saccharification by such mechanochemical treatment from the perspective of size, the process is as follows. The saccharification reaction of cellulose by cellulase is the breaking of the bond between glucose with length of $1 \mathrm{~nm}$ or less at the active site of cellulase, a protein. As a first step, cellulase must adhere to cellulose. This means cellulase first adheres to the microfibril, since cellulose is basically a microfibril. Cellulase is about $5 \mathrm{~nm}$ as a sphere, but the wood powder obtained by dry milling is much bigger. The wood powder obtained by the general dry mechanochemical treatment is about $10 \mu \mathrm{m}$ even if the processing condition is optimized, and hardly any submicron or nanosize wood powder is produced even by prolonged processing ${ }^{[9]}$. The reason is because the aggregation of particles occurs when the wood is finely broken down in the mechanochemical treatment, and the particle size may increase depending on the material.

Another point from the size perspective is the crystallinity of cellulose. The evaluation is done in most cases by powder x-ray diffraction. As mentioned in section 2.1, the cellulose crystal is a microfibril, and the amorphization of cellulose is simply looking at the disarrangement of the hydrogen bond with length of $1 \mathrm{~nm}$ or less in the region of $3 \sim 5 \mathrm{~nm}$ or less. The change of cellulose from crystal to amorphous is a change in a smaller region, considering the size of cellulase. While there are many reports on the enzymatic saccharification of cellulose, the reaction mechanism from the size perspective of cellulose and cellulase had not been clarified.

Figure 3 is a summary of the points of the conventional pretreatment technologies from the size perspective. According to the knowledge so far, the destruction and fiber production of wood structure at the level of tens of $\mu \mathrm{m}$, the compositional change by decomposition and removal of hemicellulose and lignin, and the change of crystallinity at the nano level were considered important. There were hardly any discussion for the several $\mathrm{nm}$ region at the initial phase of the enzymatic saccharification reaction (adsorption of cellulase to cellulose microfibril), and there was not much viewpoint from the microfibril side.

From the above, concerning the factors that improve the enzymatic saccharification of wood through mechanochemical treatment, we thought some guide can be obtained to construct the optimal pretreatment technology by carefully analyzing from various perspectives including the analysis at the microfibril level.

\subsection{Re-investigation of classic technology}

For the mechanochemical treatment of wood, we conducted basic experiments using a planetary ball mill, and investigated the relationship between the physical and chemical properties and the saccharification of the product ${ }^{[10]}$. When the mechanochemical treatment was conducted using roughly milled hardwood powder (Eucalyptus $<0.2 \mathrm{~mm}$ ), the breakdown of raw material wood powder progressed but

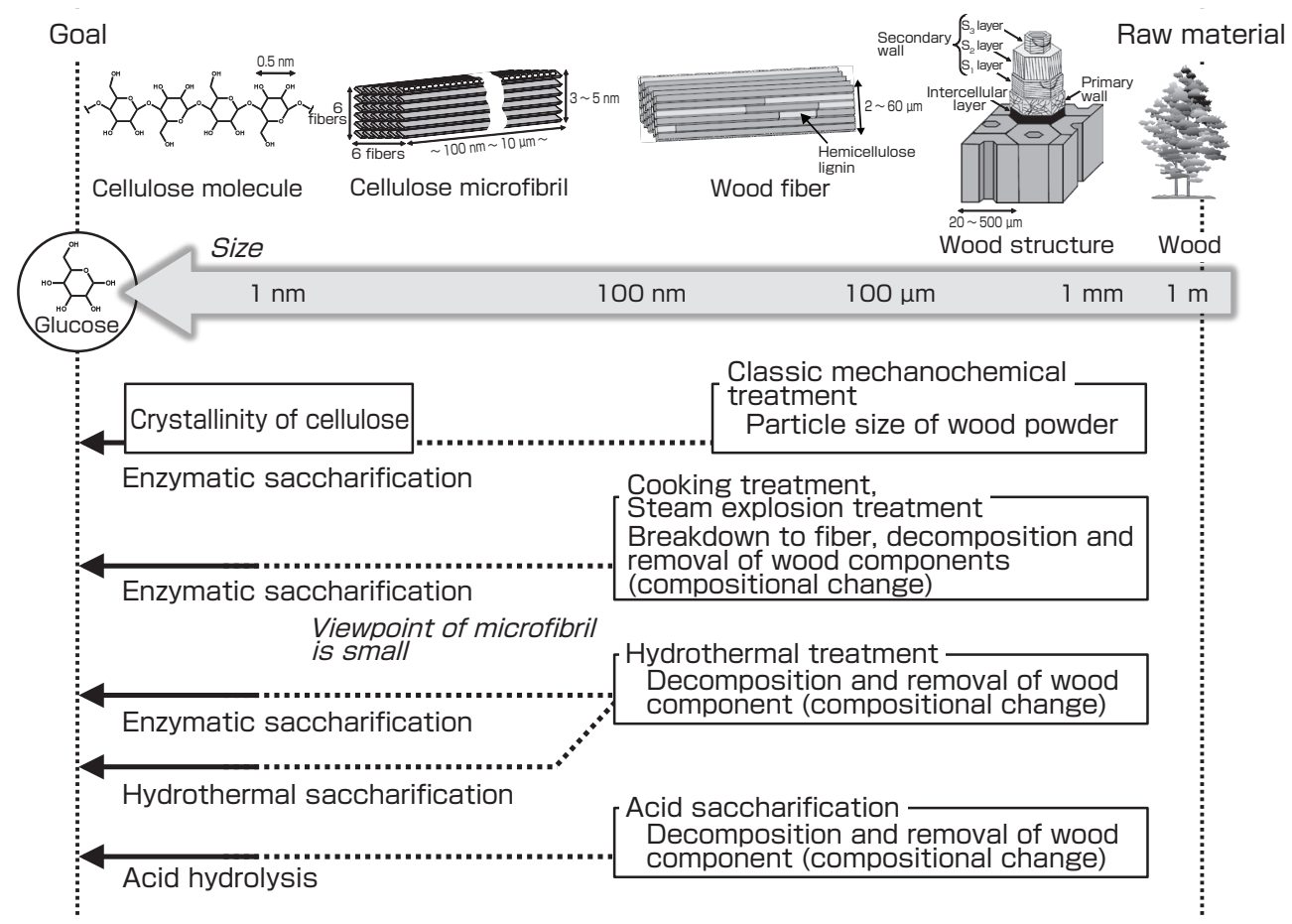

Fig. 3 Size image of (conventional) pretreatment technology for enzymatic saccharification. 
the average particle diameter of the product ceased to change from about $20 \mu \mathrm{m}$ even after milling for 1 hour or more. This indicated that the equilibrium value for the particulation and aggregation of the particles was about $20 \mu \mathrm{m}$. Looking at the enzymatic saccharification of the product, the saccharification degree was extremely low even though the untreated raw material was of fine powder of $0.2 \mathrm{~mm}$ or less. However, saccharification increased as the time of mechanochemical treatment increased. After four hours, the saccharification was 20 times or more compared to the raw material. Similar trend was seen for Douglas fir, a softwood. However, major difference in results was observed when the mechanochemical treatment was conducted using refined wood pulp (fibrous, width about $20 \mu \mathrm{m}$, length about $200 \mu \mathrm{m}$ ) in a comparative experiment. In pulp, saccharification was high even in the untreated material, and the effect of mechanochemical treatment was small (Fig. 4). From these results, it was found that enzymatic saccharification property could not be estimated from the size of the material alone.

Next, the relationship between the crystallinity of cellulose and saccharification was investigated by x-ray diffraction. As a result, in case of wood, crystallinity decreased rapidly with mechanochemical treatment whereas saccharification increased gradually. On the other hand, in the case of pulp, saccharification was high even though the raw material had high crystallinity, and crystallinity decreased rapidly by mechanochemical treatment just as in wood, but the

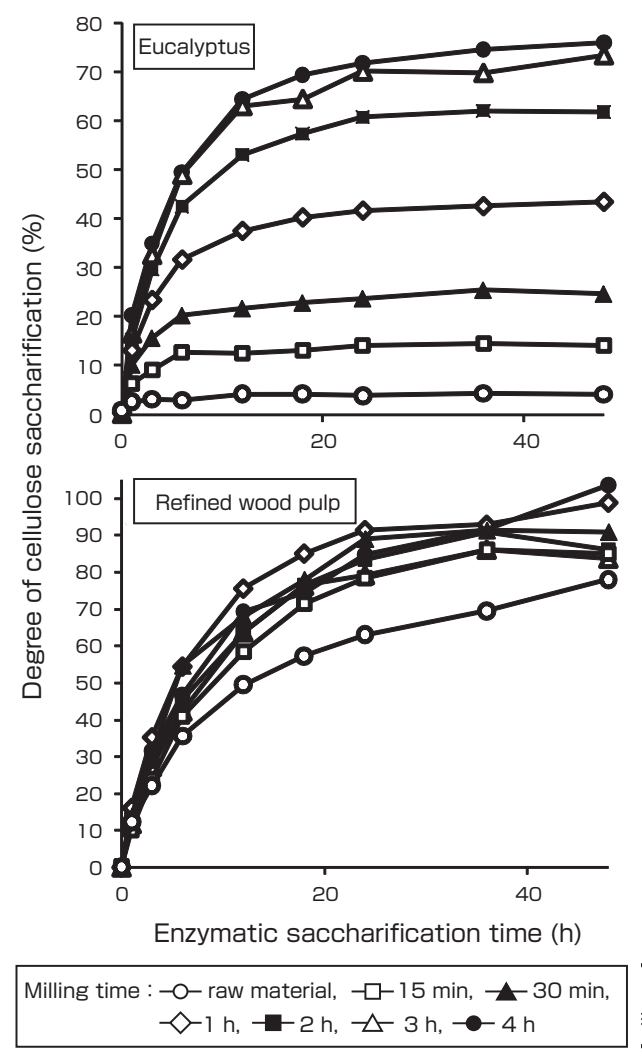

Fig. 4 Change in degree of enzymatic saccharification by milling time. relationship between crystallinity and saccharification was low (Fig. 5). The results of the mechanochemical treatment experiment conducted using woods such as Eucalyptus as raw materials did not greatly contradict the previous understanding that amorphization of cellulose was important for enzymatic saccharification. However, when pulp was used as the raw material, high saccharification was obtained with highly crystallized material, and there was a contradiction. The phenomenon where the enzymatic saccharification gradually decreased despite being amorphous when amorphized cellulose was processed for a long time by mechanochemical process had been known for some time.

From the above, it was shown that the enzymatic saccharification could not be sufficiently explained by the particle size of the mechanochemically treated product or the crystallinity of cellulose. In the above experiments, enzymatic saccharification was conducted without purification of the mechanochemically treated products, but the enzymatic saccharification degree was high. This means the enzymatic saccharification progressed even when the wood components such as lignin that supposedly inhibit enzymatic reaction remained. Moreover, from other experiments, the lignin component had high molecular weight as in an untreated wood structure even after undergoing mechanochemical treatment. Also, solid-state nuclear magnetic resonance (NMR) measurement and infrared spectrometry showed that there was hardly any transformation of wood components such as oxidization by the mechanochemical treatment.

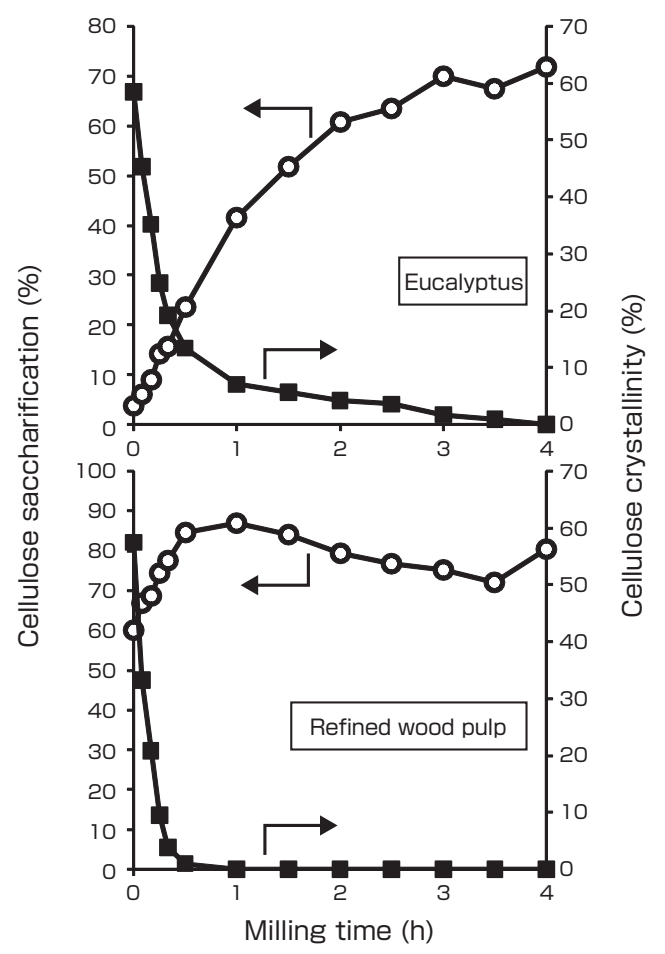

Fig. 5 Relationship between degrees of crystallinity and enzymatic saccharization. 


\subsection{Application of macromolecular chemistry techniques}

Since the aforementioned experiment results indicated that the fine breakdown of wood, amorphization of cellulose, and separation of lignin, which were the empirical rules of conventional technology, were not important in increasing the enzymatic saccharification of wood, analysis from a new viewpoint became necessary.

NMR is used frequently as an analysis tool of molecular structure in the fields of organic chemistry, and in the macromolecular field, the evaluations of mixture of molecules in complexes and molecular aggregation size (domain size) are conducted by relaxation time measurement using solid-state NMR. Relaxation time measurement is a method in which pulse signals are irradiated to a substance using the NMR device, and how fast the signal decays is measured. If the aggregation (domain) of the substance is large, the pulse signal takes more time to travel far. If the domain size is small, the pulse signal decays fast. In a case where different substances are mixed at the molecular level and placed in the same environment, the different substances will have the same relaxation time. The result of using this method to evaluate the mechanochemically treated product is as follows.

Solid-state NMR measurement was conducted at a similar moisture condition as the enzymatic saccharification reaction, and the relaxation time $\left(T_{1 \mathrm{H}}\right)$ of hydrogen atoms of the cellulose molecules was measured. As a result, the relaxation time decreased (the molecular motion increased in the macromolecular substance such as cellulose) with more mechanochemical treatment, and it was $0.05 \mathrm{sec}$. after 4-hour milling when saccharification reached maximum (Fig. 6). When the domain size was calculated from this value, it was $5.5 \mathrm{~nm}^{[11]}$. The wood became wood powder of about $20 \mu \mathrm{m}$

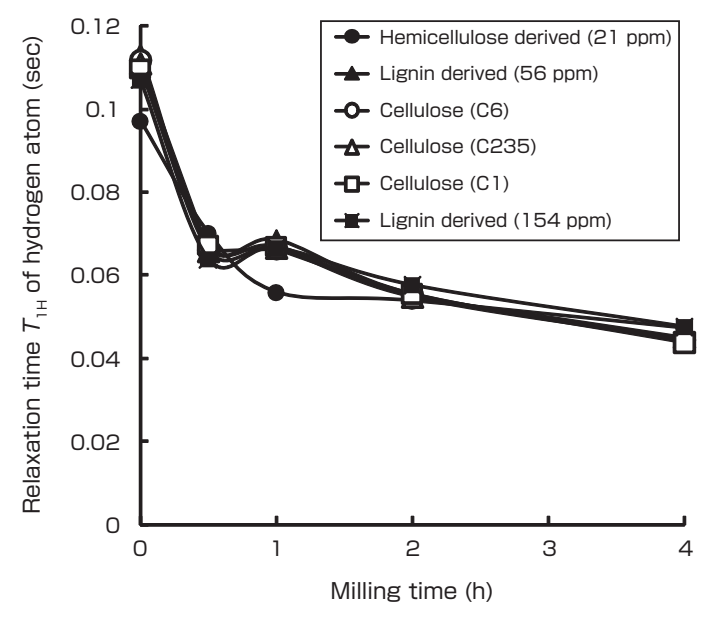

Fig. 6 Change in relaxation time $T_{1 \mathrm{H}}$ by milling time (figures in parenthesis are assignments of wood components in NMR). in appearance by the mechanochemical treatment, but this wood powder is actually composed of domains of about 5 $\mathrm{nm}$, which is much finer. Since this $5 \mathrm{~nm}$ size approached the width of the cellulose microfibril, it was hypothesized that the major factor for the promotion of enzymatic saccharification was that the microfibrils separated from each other through the mechanochemical treatment, thereby increasing the surface area where the enzymes can adsorb. The studies hereafter were based on this working hypothesis to clarify the effective mechanochemical treatment for enzymatic saccharification, and we developed a new pretreatment methodology. While the cellulose became amorphous by the mechanochemical treatment, it is thought that the orientation of cellulose molecules such as microfibril remained ${ }^{[12]}$. Looking at the size level of the fibril separation, the cellulose crystallization occurs at a lower level, and whether it is high crystallinity or amorphous is not particularly important.

\section{Microfibril treatment}

The enzymatic saccharification reaction of cellulose is a solid-liquid reaction of solid cellulose and enzyme dissolved in water. In general, to promote the solid-liquid reaction efficiently, the solid can be broken down finely to increase the contact surface with the liquid. The microfibril in the wood is the minimum unit of solid cellulose. Saccharification is expected to progress smoothly if the cellulose can be dissolved and separated into one unit of molecule, but the enzyme will easily become deactivated in a solvent that can dissolve cellulose.

Therefore, to confirm the working hypothesis based on the results obtained from the aforementioned solid-state NMR measurement, we investigated the method for actually unraveling the microfibril, which is a microscopic wood fiber. In the papermaking technology, the process called "beating" is done to increase the strength of the paper. In this process the fibers are fluffed by mechanically applying

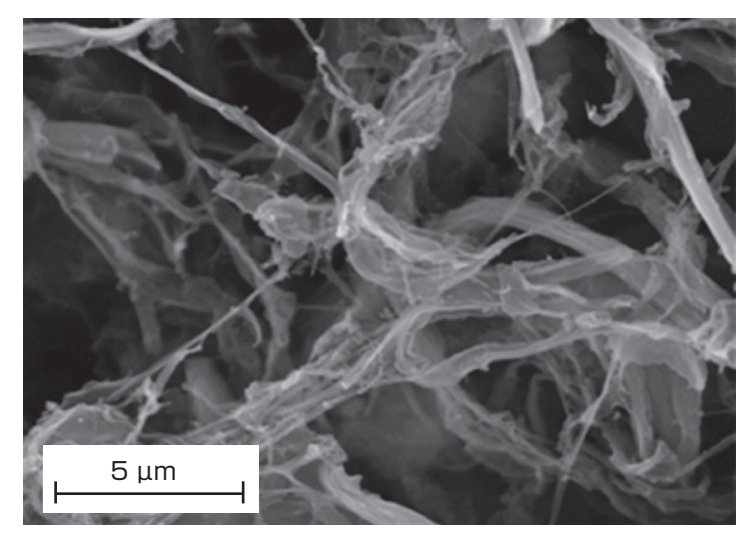

Fig. 7 Scanning Electron microscope photograph of wet mechanochemically-treated product. 
shear force to the water-dispersible slurry of pulp. The water enters the minute gaps of pulp fiber by shear force, acts like wedges to unravel the fibers, and the surrounding water prevents the aggregation of the fluffed fibers. We thought the wood could be broken down to microfibrils by using some treatment similar to the beating process. Therefore, as basic experiment, wet mechanochemical treatment was done using the ball mill with wood powder dispersed in water at 20 times the quantity by weight, and we obtained some highly viscous, creamy product. The product was dried and observed under the scanning electron microscope. It was found that the microfibril formation progressed, and fine fibers of $100 \mathrm{~nm}$ or smaller, some of $20 \mathrm{~nm}$ at the narrow parts, were produced (Fig. 7). The degree of cellulose crystallinity was studied by x-ray diffraction, and it was found that the same degree of crystallinity was maintained as the raw material. Since the microfibril itself is the crystal body of cellulose, the retention of crystal structure indicated that the microfibril could be unraveled without much damage. Although this wet mechanochemical product (microfibrilized substance) had high crystallinity, degree of saccharification was $70 \%$ or above. This result showed that decreasing the crystallinity of cellulose was not important to increase the enzymatic saccharification, but it was more important to increase the surface area with which the enzymes could react by separating the microfibrils from each other. While the concentration of solids was about $5 \%$ in the wet mechanochemical product, the cellulose microfibril with specific gravity of 1.5 was dispersed without settling out as the water molecules were retained around it. Therefore, space where the enzymes could act freely was formed around the microfibrils. Since the main residue of the saccharification experiment were large fiber structures in the samples with insufficient wet treatment and low saccharification, it was found that the enzymatic saccharification could be increased greatly if the wood were thoroughly broken down into microfibrils.

As presented above, the working hypothesis that the separation of microfibril was effective for enzymatic saccharification based on the solid-state NMR, was proven by actually creating microfibrils from wood by the wet mechanochemical treatment. The crystallinity of cellulose was not important in this pretreatment. In section 4.1 , the refined wood pulp had high degree of enzymatic saccharification while it was highly crystallized, but in this case, it was thought that the microfibrils separated from each other in the refining process of breaking down and removing the hemicellulose and lignin, and the surface to which the enzymes could approach and react increased. The wood structure is an assembly of microfibrils, and the assembly force is a weak bond such as a hydrogen bond. Therefore, by using the water molecule like a wedge in the wet mechanochemical treatment to break the hydrogen bonds between the microfibrils that keep them together, the microfibril building blocks can be disassembled easily. This method is not troublesome from the wood chemistry perspective.

\section{Microfibrilization technology for practical application}

\subsection{Investigation of continuous and mass treatment method}

The ball mill used in the fundamental experiment for the microfibrilization treatment is useful for experiments with small amounts of samples, but is done as a batch process, so it is not practical in terms of upsizing and cost reduction. Therefore, we considered a treatment method where shear force and pressure could be applied to the raw material as a wet method. We decided continuous and mass production would be possible using a disk mill that had a similar milling mechanism as a stone mortar.

We used a disk mill (Supermasscolloider, Masuko Sangyo Co., Ltd.) to repeatedly mill the wood powder slurry (wood powder concentration $5 \mathrm{wt} \%$ ), and found that the microfibrilization progressed as in the ball mill, and the enzymatic saccharification of the product greatly increased. In the disk mill treatment, the upper and lower disks were set at about $10 \mu \mathrm{m}$ distance, and the processing efficiency was 10 20 times or higher than the ball mill. However, in the disk mill, there was a dependency on the wood species that was not significant in the ball mill. In some cases, the hardwood did not microfibrilize sufficiently and the saccharification did not improve compared to softwood. This was because the milling energy was smaller in the disk mill compared to the ball mill. Therefore, treatment to weaken the wood structure was necessary before the disk mill treatment.

\subsection{Combination treatment for weakening the wood structure}

The origin of wood strength is the robust layer structure as mentioned before. Although the microfibrils are held together by weak bonds such as hydrogen bonds, separation to microfibril units is not easy due to its board-and-hoop layer structure. Therefore, we thought the wood structure would weaken and the disk mill treatment could be conducted effectively by first destroying the structure that corresponded to the hoop of the tub to increase the water permeability, and then removing the hemicellulose that glued the microfibrils together.

The method for destroying the structure that corresponded to the hoop was preliminarily investigated using the ball mill. After conducting dry mechanochemical treatment of the raw wood powder for a certain time, water was added for wet treatment, and the microfibrilization and saccharification of the product were studied. As a result, when the time of dry mechanochemical treatment was 15 
min or less, no major change was seen, while saccharification significantly improved in the wet-treated product obtained after dry treating for $20 \mathrm{~min}$ or more. When the dry mechanochemically treated product was observed by a scanning electron microscope (SEM), it was found that the large wood structure of the raw wood powder was almost entirely destroyed by the 20 -min dry mechanochemical treatment. Since the ball mill process was not practical for destroying the hoop structure, we considered the method that had relatively large milling energy and enabled mass processing, and found that wet cutter mill was effective. The wet cutter mill we employed (Micro-Meister; Masuko Sangyo Co., Ltd.) had powerful shear force with a fixed blade and a rotary blade that rotated at ultra high-speed of 10,000

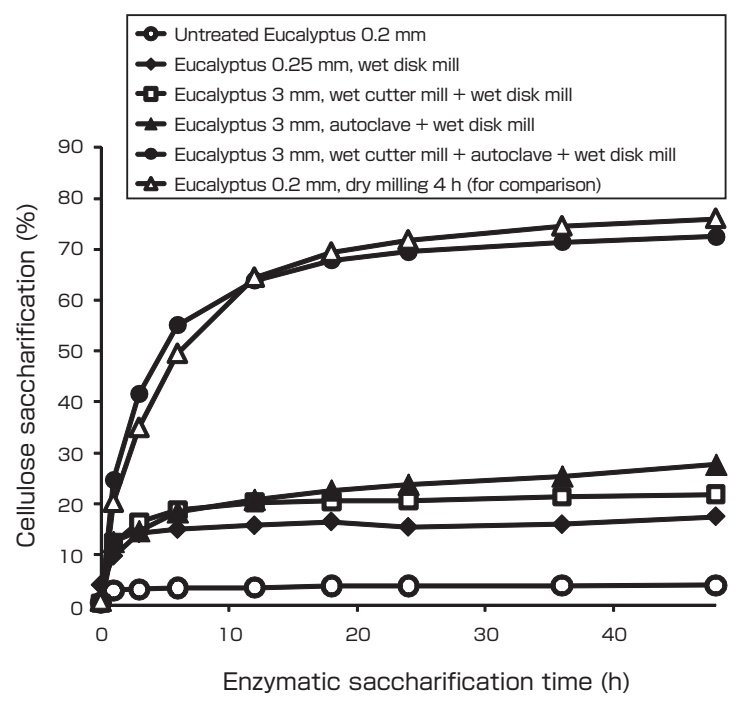

Fig. 8 Increasing efficiency of disk mill treatment by combination treatment. rpm or more, and was capable of instantly breaking down the raw material dispersed in water to $1 \mathrm{~mm}$ or less. In this case, water fluidized the milled product, prevented stagnation, and promoted the break down efficiently. Also, the water that permeated the wood structure during the process also worked effectively for the following autoclave and disk mill treatments.

Next, we conducted the hydrothermal treatment using the autoclave as a method for reducing the adhesion effect of hemicellulose. The hydrothermal treatment allowed the selective hydrolysis of hemicellulose component according to the temperature condition ${ }^{[13]}$. Investigating a combination treatment method that combined the wet cutter mill and autoclave treatments, it was found that efficient pretreatment was possible regardless of the wood species by conducting the autoclave treatment $\left(135^{\circ} \mathrm{C}\right)$ after conducting the wet cutter mill treatment ( $1 \mathrm{~mm}$ or less) on the roughly milled raw wood powders, and then conducting the disk mill treatment as the final stage. When the Eucalyptus raw material was roughly milled to $3 \mathrm{~mm}$ or less by the combined treatment, the degree of saccharification was 4 times or more compared to the case when wood powder $0.25 \mathrm{~mm}$, which was finer, was treated with the disk mill only. Approximately the same saccharification was obtained as for the long dry ball milling (Fig. 8).

\subsection{Mechanism of improvement of enzymatic saccharification by combination wet mechanochemical treatment}

The mechanism by which the enzymatic saccharification improves due to the aforementioned combination wet mechanochemical treatment is thought to be as follows

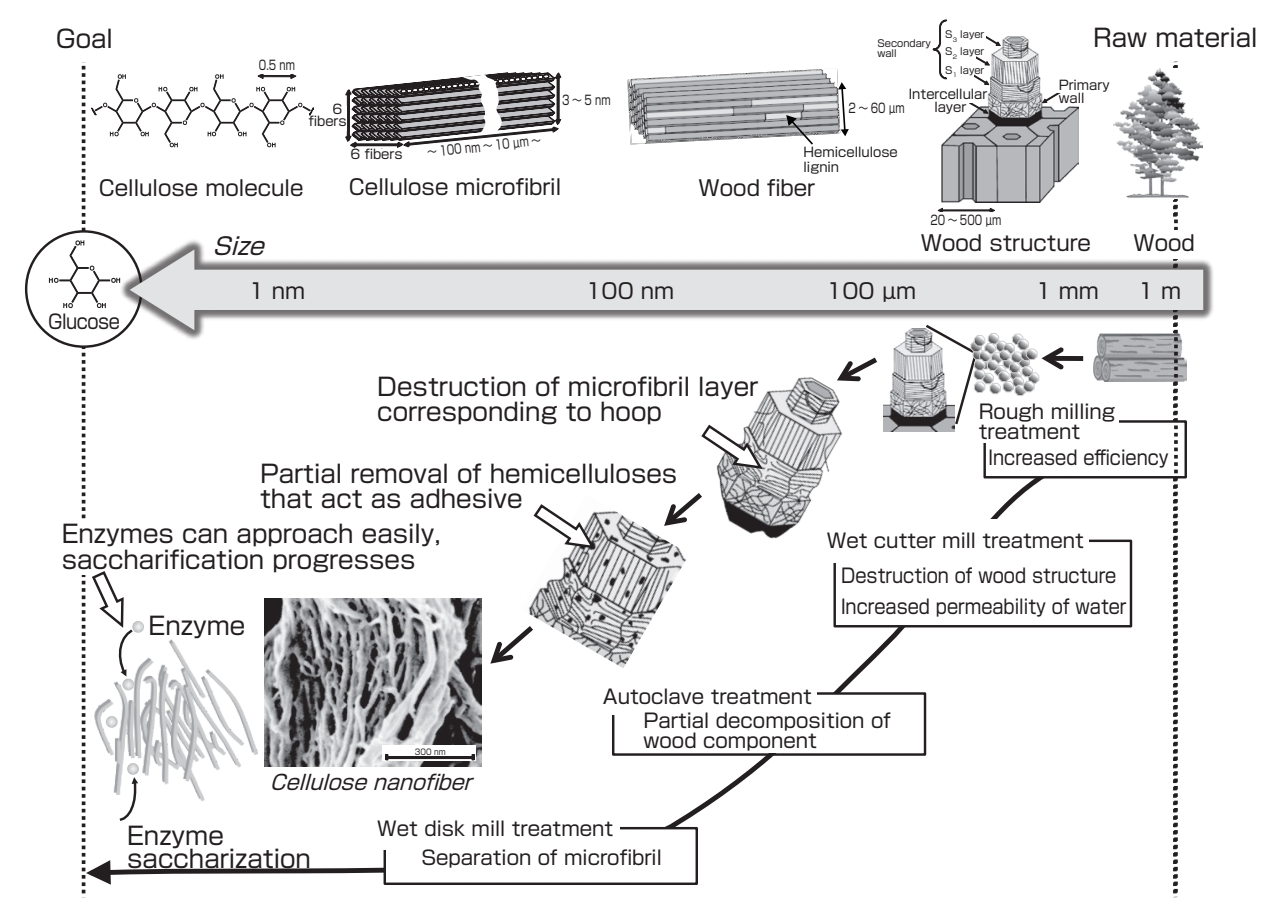

Fig. 9 Size image of combination wet mechanochemical treatment. 
(Fig. 9). The cell structure of wood is $20-500 \mu \mathrm{m}$, and the wood structure is destroyed by the powerful shear force of the wet cutter mill. The microfibrils that correspond to the hoop are partially broken. Next, the hemicellulose that glues the microfibrils together is partially hydrolyzed (few $\%$ of all hemicelluloses) by the autoclave treatment. When the autoclaved product was observed at high magnification by SEM, a large number of pores of tens of nm were observed. These pores are thought to be the holes after the hemicellulose dropped out. As the wood structure weakens after these steps, the structure can be unraveled into microfibrils easily by the final disk milling, and the enzymatic saccharification is improved greatly.

So far, the explanation was centered on the mechanism for the improvement of enzymatic saccharification of the cellulose component. However, in bioethanol manufacturing, the saccharification of hemicellulose is also important to increase the yield of sugar as fermentation material. In the wood structure, hemicellulose covers the surface of the cellulose microfibril, and in our pretreatment process where the microfibrils are separated from each other, the saccharification of hemicellulose progresses alongside the saccharification of cellulose. Since drastic chemical reaction does not take place in our pretreatment, the structure of lignin does not change from the structure in the untreated wood. After enzymatic saccharification, the lignin remains as residue.

\section{Advantage of the mechanochemical treatment}

\subsection{Cost reduction of enzymatic saccharification}

As mentioned earlier, the disk mill treatment has high efficiency and relatively low cost. However, compared to the

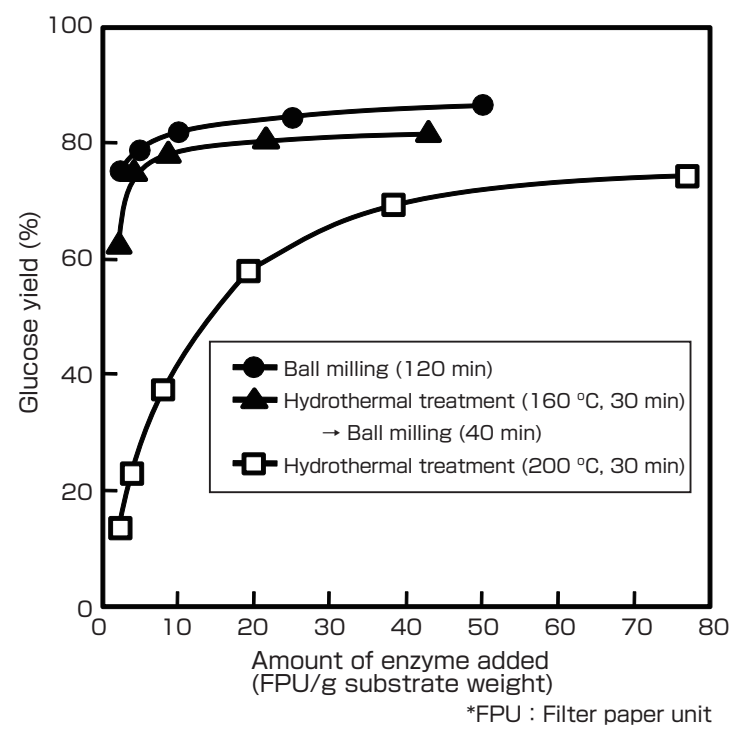

Fig. 10 Relationship between amount of enzyme added and saccharification by different pretreatment. simple heat treatment, dramatic cost reduction is not possible since the driving power of motors cannot be recovered or recycled. Yet, the advantage of the mechanochemical treatment is that the costs in other processes of the bioethanol production can be reduced greatly. In the mechanochemical treatment, enzymatic saccharification and fermentation progress efficiently without the chemical treatments such as lignin removal. Also, dependency on raw material is low, and the process can be applied as the pretreatment of hardwoods, softwoods, straws, and others. The greatest advantage is the reduction in the cost of enzymes such as cellulase, which in some cases dominate over half the cost of bioethanol production. The mechanochemically treated products can be sufficiently saccharified with relatively small amount of enzymes. Figure 10 shows the relationship of the amount of enzymes and the degree of saccharification of the products of various pretreatments ${ }^{[14]}$. Compared to the mechanochemical treatment, the degree of saccharification falls greatly when the amount of enzymes is small in the $200{ }^{\circ} \mathrm{C}$ hightemperature hydrothermal process. This phenomenon is thought to be due to the production of inhibitor from the transformation of wood components in the high-temperature hydrothermal process. Similar results are expected in the acid treatment that follows the similar reaction as the hydrothermal treatment. Relatively low temperature $\left(160{ }^{\circ} \mathrm{C}\right)$ treatment reduces the time for mechanochemical treatment, and saccharification progresses even with small amount of enzymes.

\subsection{Comparison with conventional technology}

The comparison of the characteristics of the conventional technology against the pretreatment process for enzymatic saccharification we developed is as follows (table at bottom of Fig. 2). The degree of saccharification of the treated product is about the equivalent to that of the classic mechanochemical treatment. Advantages are: there is no need of advanced reaction control as in the chemical process; and since only water is used for the process, recovery of chemicals is not necessary and waste liquid management is easy. With the same amount treated, the electricity consumed by our process is about 1:10 20 or less of the conventional ball mill process. The autoclave treatment also has low energy consumption since it is not a high-temperature high-pressure process, and the equipment does not have to be high-pressure resistant. As mentioned earlier, the dependency on raw material biomass species is low. Also, our pretreatment process is basically a wet process, and the raw material does not have to be dried. Raw materials with high water content can be used as is. In the concentrated sulfuric acid saccharfication, it may be necessary to dry the raw material to prevent heating due to dilution of the sulfuric acid, and therefore, the efficiency is poor.

The pretreatment process we developed overcomes the various issues of the conventional technology, and can be applied without loosing the advantages. The new issue is 
that since the wet cutter mill and the disk mill used in this $\mathrm{R} \& \mathrm{D}$ are precision mills, they cannot be readily upsized. However, since our pretreatment process is similar to the mechanical pulping technology of papermaking, we believe up-scaling and practical utilization can be done by applying and developing the papermaking technology.

\section{Summary and future developments}

At AIST, we built an integrated manufacturing miniplant (capable of treating $200 \mathrm{~kg}$ of diverse biomass at once) that incorporates our pretreatment process and the saccharification and fermentation processes. Here, extraction of issues in individual elemental technologies and process continuity, issues on biomass species, and economical feasibility assessments are being done. Currently, R\&Ds are conducted with companies for commercialization using largescale processes, and papermaking technologies are being incorporated.

In the practical application of bioethanol production technology, high added value for residues and byproducts is also important. The microfibrilized product obtained by the pretreatment of wood is called cellulose nanofiber since it is a nanosize fine fiber. Compared to steel of the same size, its weight is $1 / 5$ lighter and the strength is 5 times greater. Currently, the development of lightweight high-strength material using this property is being developed ${ }^{[15]}$. Also, application to optical materials and cosmetics is being studied. Some are already in use as filter materials and food additives.
In our pretreatment process, no major change in the molecular structure of the wood components occurs. Therefore, the lignin residue after the enzymatic saccharification is unlike the black liquor (broken down lignin) produced as waste in the papermaking process. It is thought to contain structures similar to wood with high molecular weight. This lignin residue can be converted to polymer material and high value-added material that was impossible with lignin in the black liquor that could only be used as fuel. Extremely high economic feasibility can be attained using the cellulose nanofiber production as a common fundamental process with the production of bioethanol (main) and high value-added material (subsidiary).

Figure 11 is a summary of the flow of our pretreatment technology development. As a result of studying the conventional technology, we found that the viewpoints from the wood chemistry and cellulose chemistry of the target wood were necessary for the development of the practical pretreatment technology. Moreover, by combining the knowledge and technologies of other fields such as macromolecular chemistry and papermaking, we were able to construct a pretreatment technology based on a new concept. However, there are many unclear points about the properties and reaction mechanism of the enzymes for the cellulose nanofiber obtained by pretreatment, and state-of-the-art analyses are necessary. In the future, the technologies and knowledge of biotechnology, chemical engineering, thermal engineering, LCA assessment, and social sciences may be fused to establish a practical biofuel technology.

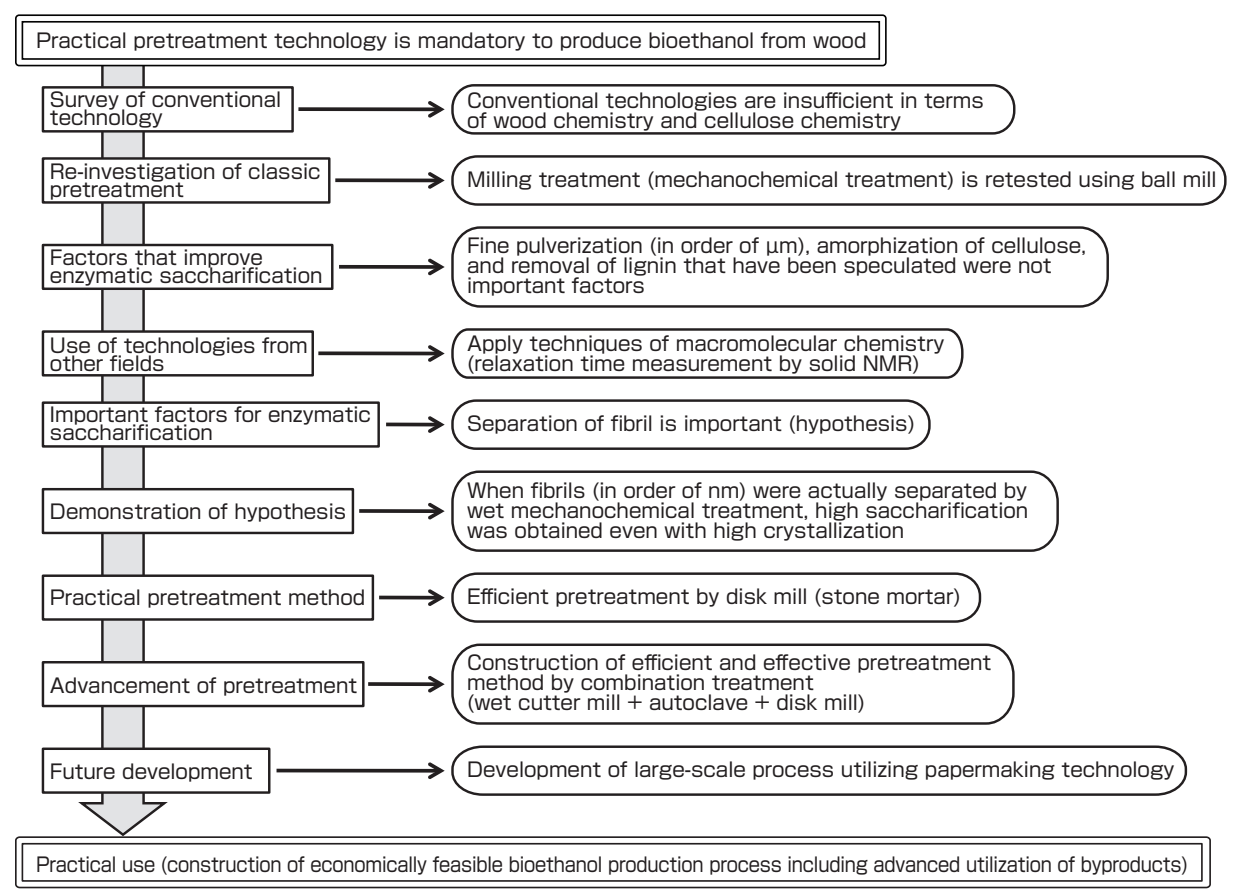

Fig. 11 Flow of pretreatment technology development. 


\section{References}

[1] E. Farrell, R. J. Plevin, B. T. Turner, A. D. Jones, M. O'Hare and D. M. Kammen: Ethanol can contribute to energy and environmental goals, Science, 311, 506-508 (2006).

[2] J. F. Saeman: Kinetics of wood saccharification - Hydrolysis of cellulose and decomposition of sugars in dilute acid at high temperature, Ind. Eng. Chem. Res., 37, 43-52 (1945).

[3] K. Shimizu: Serurosu kei busshitsu no koso kasui bunkai (Enzymatic hydrolysis of cellulose substance), Kinosei Serurosu (Functional Cellulose), 195-229, CMC Publishing, Tokyo, (1985) (in Japanese).

[4] T. Endo: Mechano-chemical preparation of novel cellulose composite, Cellu. Commun. (Journal of the Cellulose Society of Japan), 7 (2), 63-66 (2000) (in Japanese).

[5] T. Endo, F. R. Zhang and Y. Shinohara: Cellulose as molding material, Cellu. Commun. (Journal of the Cellulose Society of Japan), 9 (2), 86-92 (2002) (in Japanese).

[6] T. Endo and M. Ago: Mechano-chemical modification of cellulose structure, Cellu. Commun. (Journal of the Cellulose Society of Japan), 11 (2), 74-78 (2004) (in Japanese).

[7] T. Endo and A. Isogai, eds: Bifunsaika serurosu to purasuchikku no fukugoka (Compositing of finely milled cellulose and plastic), Serurose Riyo Gijutsu No Saisentan (Advanced Technologies of Cellulose Utilization), 298-309, CMC Publishing, Tokyo, (2008) (in Japanese).

[8] R. Tanaka, F. Yaku, E. Murai and T. Koshijima: Enzymatic degradation on finely divided wood meal of Pinus densiflora, Cell. Chem. Technol., 14, 859-868 (1980).

[9] T. Endo, R. Kitagawa and J. Hosokawa: Kikaiteki funsai niyoru serurosu sen'i no biryushi keisei kyodo (Fine particle formation behavior of cellulose fibers by mechanical milling), Kobunshi Ronbunshu (Japanese Journal of Polymer Science and Technology), 56 (3), 166-173 (1999) (in Japanese).

[10] S. Fujimoto, H. Inoue, S. Yano, T. Sakaki, T. Minowa, T. Endo, S. Sawayama and K. Sakanishi: Bioethanol production from lignocellulosic biomass requiring no sulfuric acid: mechanochemical pretreatment and enzymic saccharification, Sekiyu Gakkaishi (Journal of the Japan Petroleum Institute), 51 (5), 264-273 (2008) (in Japanese).

[11] D. Ishii, D. Tatsumi and T. Matsumoto: Effect of solvent exchange on the solid structure and dissolution behavior of cellulose, Biomacromol., 4, 1238-1243 (2003).

[12] M. Ago, T. Endo and T. Hirotsu: Crystalline transformation of native cellulose from cellulose I to cellulose II polymorph by a ball-milling method with a specific amount of water, Cellulose, 11 (2), 163-167 (2004).

[13] H. Ando, T. Sasaki, T. Kokusho, M. Shibata, Y. Uemura and Y. Hatate: Decomposition behavior of plant biomass in hotcompressed water, Ind. Eng. Chem. Res., 39 (10), 3688-3693 (2000).

[14] H. Inoue, S. Yano, T. Endo, T. Sakaki and S. Sawayama: Combining hot-compressed water and ball milling pretreatments to improve the efficiency of the enzymatic hydrolysis of eucalyptus, Biotechnol. Biofuels 1:2 1-9 (2008).

[15] H. Yano and A. Isogai eds: Serurose nano faiba fukugo zairyo (Cellulose nanofiber composite material), Serurose Riyo Gijutsu No Saisentan (Advanced Technologies of Cellulose Utilization), 258-269, CMC Publishing, Tokyo, (2008) (in Japanese).

\section{Author}

\section{Takashi Endo}

Completed the doctorate course at the Department of Chemistry, Graduate School of Science, Hiroshima University in 1992. Doctor (Science). Joined the Shikoku National Industrial Research Institute, Agency of Industrial Science and Technology in April 1992. Engaged in research for the pulverization of cellulose and wood using the milling technology

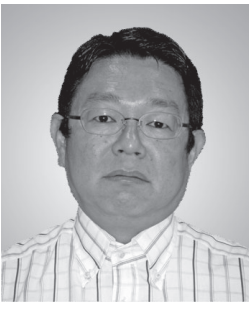
and high added-value technology. Worked mainly in the field of materials to 2003. After transferring to the current institute in 2004, has conducted R\&D for bioethanol. Leader of the research team of the Biomass Technology Research Center from 2006 to present. This paper is a summary of part of the research results for cellulose milling that has been the subject of study since joining the institute.

\section{Discussion with Reviewers}

\section{Advantages compared to conventional treatment methods (cooking, steam explosion, and hydrothermal methods)}

Question (Koichi Mizuno, Research Institute for Environmental Management Technology, AIST)

In the conversion process from wood to ethanol when limited to enzymatic saccharification, Fig. 2 shows the comparison of the developed method combining the wet cutter mill, autoclave, and wet disk mill treatments to other conventional methods (cooking, steam explosion, and hydrothermal treatments). Please indicate quantitatively or qualitatively how much the advantages and strengths have been progressed and to what degree the disadvantages and problematic issues have been overcome.

\section{Answer (Takashi Endo)}

The advantage of the combination treatment we developed is that a pretreated product with high degree of saccharification can be obtained with low environmental load and with no dependency on wood species. Compared to ball milling, the conventional technology, the consumption energy is 1:10 20 or less. Also, in our pretreatment method, saccharification occurs with a small amount of enzymes, so the cost of enzymes can be reduced. Woods with high moisture content immediately after lumbering can be used as raw materials. Since there is no use of chemicals in large amounts, I think there is high potential for commercial bioethanol production at the site of raw material harvesting in the future (such as in Southeast Asia). At the Biomass Technology Research Center where I work, there is a research team that specializes in assessing economic feasibility. We conducted some economic projections at large-scale commercial levels (raw material 1500 ton/day) using the data for pretreatment and saccharification-fermentation processes that we actually conducted in the lab. As a result, there was the possibility for decreasing the cost of bioethanol final product to a level lower than the NEDO's concentrated sulfuric acid method that was considered to have the lowest cost (sufficiently accurate data for economic feasibility are not disclosed for other methods). Since the combination treatment is a multi-step treatment, the cost of the facilities may be higher, but the facility depreciation can be actually the same as the concentrated sulfuric acid method. For example, there is a hydrothermal process in our pretreatment, and the temperature needed is about $150{ }^{\circ} \mathrm{C}(0.48 \mathrm{MPa})$ which will not be subject to the strict regulation of the High Pressure Gas Safety 
Law (1 MPa or higher), so the maintenance of the plant will be easy. In the future, we shall optimize the process further, and investigate ways to enhance efficiency by using chemicals that is low cost, has low environmental load, and does not affect the following saccharification and fermentation.

\section{General use of biomass}

\section{Question and comment (Koichi Mizuno)}

In this research, using the wood as starting materials, the fermentation process of cellulose and hemicellulose to ethanol through enzymatic saccharification was shown, as well as the ways of manufacturing materials such as polymers from lignin. This may lead to the biomass refinery concept that may replace the conventional refinery using crude oil. What do you think is the effective R\&D strategy to promote the biomass refinery?

Answer (Takashi Endo)

Although there is a long history of research for use of lignin, there has been no definitive technology or product. However, when bioethanol production is commercialized, like in our technology, new lignin different from the one in the conventional papermaking process is produced in large amounts. Looking at the current petrochemical products, crude oil can be used without loss from asphalt and gasoline to plastic. In the future biomass refinery, I think the development of a technology to utilize the wood components without loss and the construction of a social system where they can be utilized are important. To achieve this, I think it is important to create some characteristic products that can only be achieved with biomass products, rather than creating alternatives to petroleum products. To do so, it is necessary to accurately understand the basic properties of cellulose nanofiber and residue lignin obtained from pretreatment, by using analytical methods, and to establish an innovative product realization technology based on the new knowledge obtained and the theories and principles that are already known. 